\title{
Mudanças, em curso e futuras, dos sistemas educacionais: Desafios e marcas dos anos 1960 aos anos... 2030! ${ }^{1}$
}

\author{
Mutations, en cours et à venir, des systèmes éducatifs. \\ Défis et repères des années 1960 aux années... $2030 !^{23}$
}

Raymond Duval Professor Emérito da Université du Littoral Côte d'Opale/França

\section{Tradução: Méricles Thadeu Moretti \\ PPGECT/UFSC \\ mthmoretti@gmail.com}

Desde os seus desenvolvimentos institucionais, no século XIX, os sistemas educativos, em todos os países, experimentaram uma série de mudanças e reformas na organização dos currículos, das disciplinas, dos instrumentos pedagógicos utilizados, dos exames e das avaliações. Mas, desde 1960, pode-se falar de uma verdadeira mudança, independente daqueles que decidem, sejam eles especialistas científicos, pedagogos ou políticos. E isso levou a uma ruptura completa com o que a escola dos níveis fundamental e médio foram e ainda são.

\section{Desafios atuais e futuros}

Esta ruptura pode ser vista sob dois aspectos, qualquer que seja o sistema educativo: as exigências relacionadas ao "saber" que um sistema educativo deve promover a sua aquisição; o professor em sala de aula.

1. As exigências tocantes aos "saberes" são formuladas em termos de objetivos e de conteúdos a serem aprendidos. Essas exigências surgem por conta das necessidades da sociedade em matéria de educação e formação. Duas exigências parecem tornar-se

\footnotetext{
${ }^{1}$ Conferência proferida na Faculdade de Ciências Sociais e de Ciências da Educação da Universidade de Chipre em 20 de novembro de 2014.

${ }^{2}$ Conférence donnée à la Faculté des Sciences Sociales et des Sciences de l'Education de l'Université de Chypre, le 20 novembre 2014.

${ }^{3}$ Dans l'annexe, on trouve la Conférence en français.
} 
primordiais e, o que eu gostaria desde já sublinhar que são, de um estrito ponto de vista educativo, fortemente em oposição.

1.1 A primeira corresponde ao que chamarei de filosofia das enquetes internacionais PISA (Programme for International Student Assessment). Ela é perfeitamente expressa pelo Diretor de l'OECD (Organisation for Economic Co-operation and Development), responsável pela avaliação do "nível dos alunos" aos 15 anos de idade em 65 países. Eu o cito em uma entrevista recente (Le Monde, domingo, 30 de agosto de 2014, p. 6): "O mundo não se importa com o que você sabe, interessa-se com o que você sabe fazer". Dito de outro modo, não são mais os conhecimentos que contam, mas o que você pode por em prática em situações concretas. Vocês reconhecem, aqui, uma abordagem por objetivos em termo do "saber-fazer" e das "competências". Esta exigência corresponde a uma dupla demanda social referente ao mundo do trabalho: que depois de formados os jovens encontrem trabalho e, os serviços públicos, pessoal qualificado.

1.2 A segunda exigência corresponde ao o que eu chamaria de desenvolvimento da capacidade de refletir e de aprender por si mesmo contra a inércia do que se sabe e a ansiedade diante do desconhecido de uma novidade que podem ser traduzida por expressões do tipo "a gente não aprendeu isso", “a gente nunca viu isso", "como é que é pra fazer?". Aqui, não são mais os saberes e os saberes-fazer que importam, mas o que lembra o que se chama de um termo kantiano, tornado hoje, ao mesmo tempo, um objetivo e um meio: a autonomia do aluno. Um slogan dos anos 1960-1970, o resume: "aprender a aprender". Acentua-se, agora, entre outras coisas, mais nos métodos e procedimentos do que nos conteúdos. Esta exigência é, evidentemente, mais antiga, mas tornou-se imperativa com a aceleração das descobertas científicas em que a noção de "progresso" dá agora uma ideia falsa, e das transformações tecnológicas dos instrumentos. O resultado é uma defasagem crescente entre, por um lado, os conhecimentos ensinados e, por outro lado, os conhecimentos reais que estão em expansão em diferentes domínios da pesquisa e suas aplicações em todos os setores da atividade profissional. Essa defasagem impõe aprender não só na escola, mas ao longo de toda a vida. Hoje estamos em uma situação em que se deve aprender mais ao longo da vida do que durante o ensino médio, ou mesmo, universitário. Assim, a palavra "formação" substitui, de certo modo, a palavra "educação". É a partir dos anos 1970 que se começa a organizar a "formação permanente", a "formação continuada", a "formação de adultos". 
2. Há um questionamento em relação ao par "sala de aula-professor" que até o presente momento foi o par motor dos sistemas educativos. Tal questionamento, em primeiro lugar, focalizou a forma de ensinar. Isto se traduziu por um questionamento das aulas expositivas em benefício dos "métodos ativos". Dito de outro modo, a função de ensinar não é mais transmitir diretamente os saberes, mas organizar ou de "gerir" o trabalho dos alunos em sala de aula para que eles possam adquirir os saberes e para "dar sentido" a essas aquisições.

Mas, o questionamento mais radical toca atualmente a sala de aula, quer dizer, o princípio mesmo de uma organização comum e uniforme do trabalho por um grupo de 20 a 40 alunos. É importante lembrar que esta organização, comum, não se traduz somente em um programa de conteúdos a serem aprendidos, mas na elaboração de sequências de atividades e de "engenharia didática" para a aquisição de cada um dos conteúdos.

Deste modo, as pesquisas sobre o "ensino" de matemática e sobre a formação de professores, começaram, no âmbito do $\mathrm{IREM}^{4}$, por ocasião da Reforma da matemática moderna, desenvolveram-se, sobretudo, em se centrando na organização do trabalho em sala de aula. Ora, é este princípio mesmo que aparece agora inadaptado e ultrapassado. O desaparecimento da sala de aula é inevitável e previsível para... 2030! É esta a conclusão que um fórum de 645 "especialistas mundiais de educação" chegou (Le Monde, 10 de outubro de 2014, p. 10), uma vez que há uma tendência à individualização do ensino, o que resulta, ipso facto, conteúdos mais individualizados que vão suplantar qualquer organização comum de ensino.

O que é importante, em relação a esta tendência, não é de saber se ela vai levar ao desaparecimento parcial ou completo da sala de aula, mas de ver porque as mudanças tecnológicas do ambiente, por um lado, e o fracasso dos sistemas educativos, por outro lado, impõem uma individualização do ensino.

2.1 A mais poderosa mudança tecnológica é a "digital" que universalizou, em todos os domínios, a "ação interativa com um monitor". Um comando, reduzido a um clique, em uma lista de opções ou o toque de um ícone, exibe, no monitor, as imagens ou informações que se quer. Essa mudança permitiu uma dupla individualização:

\footnotetext{
${ }^{4}$ N. Trad. IREM (Instituts de Recherche sur l'enseignement des Mathématiques) são institutos espalhadas em diversas regiões da França que desenvolvem pesquisas sobre o ensino e aprendizagem da matemática.
} 
- qualquer um pode ter acesso individual direto e imediato a todo o conhecimento e a todas as trocas possíveis, a partir do desenvolvimento da Internet e web sites (1989-1994);

- Qualquer um pode usar, individualmente, sem qualquer treinamento prévio, as ferramentas de tratamento da informação (calculadoras, tratamento de texto, programas computacionais de construção de gráficos, de figuras, etc.).

2.2 Os limites e falhas dos sistemas de educação aparecem, inicialmente, com os insucessos dos alunos e da maneira como eles os experimentam. Isto se manifesta por uma falta de motivação e mesmo pela marginalização de uma parcela cada vez mais não desprezível de alunos, especialmente, a partir das quatro séries finais do ensino fundamental. Na França, diz-se de estudantes que "abandonam". Isso se traduz, não somente por meio de enquetes PISA, mas por meio de avaliações, sejam elas feitas em nível nacional ou em uma escala mais reduzida como no âmbito de pesquisas científicas. Sem entrar na discussão da pertinência das questões formuladas e na maneira de como combiná-las para determinar o que é adquirido, todas as enquetes e todas as avaliações podem ser lidas segundo o princípio da garrafa meio vazia ou meio cheia, ou da garrafa a um terço cheia ou a dois terços vazia. Tudo depende do que se quer concluir a respeito do sucesso ou fracasso de um sistema de educação ou de uma experiência de ensino.

\section{Marcos para a educação: o caso exemplar e crucial do ensino de matemática}

Eu mencionei os desafios, agora gostaria de indicar alguns pontos de referência para uma educação que prepara as crianças e os jovens para um mundo em plena transformação tecnológica, cultural e social. Por isso, vou tomar o exemplo do ensino da matemática. Por quê essa escolha? Antes de tudo, eu trabalho com o ensino de matemática desde a criação do IREM em 1969 e pude seguir de perto tanto dos alunos quanto dos professores, as evoluções e mudanças que evoquei. Mas há duas outras razões mais sérias que atingem os objetivos de dos sistemas educativos. A matemática desempenha um papel chave na aprendizagem de outras disciplinas e, sobretudo, a compreensão da matemática é pedagogicamente fundamental e insubstituível para o desenvolvimento das capacidades mentais. É sobre este papel de formação da mente que eu gostaria de insistir e, para isso, vou reter dois aspectos de compreensão da matemática em relação aos desafios mencionados. 
2.3 A matemática é um tipo de conhecimento em que: ninguém pode realmente entender no lugar de outro; não se pode nunca repetir ou reproduzir o que foi aprendido, o que é encontrado em uma web site, ou aquilo que outras pessoas mostram. Isso significa duas coisas que podem parecer preocupante de um ponto de vista educativo tradicional:

(1) Diferentemente de outros conhecimentos, o conhecimento matemático não é comunicável. Mais exatamente, pode-se compreender os resultados científicos sem ter que fazer os procedimentos científicos que possibilitam descobri-los. Mas, os resultados matemáticos não são compreensíveis enquanto não se puder refazer, por si mesmo, os passos que conduziram chegar a eles. Isto quer dizer que as explicações de outros (professor ou aluno) não conseguirão fazê-lo compreender. É apenas quando se consegue fazer por si mesmo que se pode compreender matemática.

(2) Você não pode usar o conhecimento matemático aprendido (fórmulas, por exemplo) para resolver problemas da realidade ou problemas científicos se você não entendeu, por si mesmo, como se pensa e se trabalha em matemática.

Em outras palavras, a matemática é um tipo de conhecimento cuja prática permanece eminentemente individual, mesmo que seja possível uma comunicação objetiva e científica, mas unicamente, entre aqueles que já entendem a matemática! Ora, se a gente não a compreende por si mesmo, a matemática é apenas um jargão, sequências de números às cegas ou gráficos ilusionistas que aparecem nos monitores? A compreensão matemática inscreve-se nesta exigência de individualização do ensino (ou seja, um ensino que não seja centrado na organização em classe) que se impõe como uma necessidade dos sistemas educativos.

2. 2. A matemática é, desde o princípio, a experiência de uma autonomia intelectual completa. Por quê? Em matemática, é preciso fazer por si mesmo para poder compreender (ver 1.1). Ora, a matemática apresenta duas características cognitivas e epistemológicas que a diferenciam de todos os outros tipos de conhecimento e atividade; ela se desenvolve de forma independente de qualquer fonte externa de informação, unicamente por meio de transformações de representações produzidas em sistemas semióticos comuns (linguagem natural e diagramas) e em sistemas semióticos especializados (sistemas numéricos, gráficos, figuras geométricas). E é, graças a essas transformações de representações semióticas, que a atividade matemática dá um poder ilimitado de exploração e é gerador de novos 
conhecimentos. Em outras palavras, a compreensão matemática baseia-se na autonomia de uma atividade que é puramente intelectual (o que não significa meramente "conceitual"), que deve ser praticada individualmente. Esta autonomia, que é a autonomia do pensamento, se manifesta de duas formas:

(3) Você não precisa pedir a alguém (professor ou aluno) para saber se o que você encontra é verdade ou não, ou se um resultado é certo ou errado. Você pode explicar como fazer para obter um resultado e também por que funciona ou por que não funciona;

(4) Você não precisa perguntar a alguém "o que é preciso fazer?" diante de um problema a ser resolvido, você pode tomar iniciativas e buscar o que irá levar à solução. Você terá confiança na sua capacidade de análise e iniciativa para enfrentar algo desconhecido ou novo.

Isso pode ser resumido no seguinte círculo didático: para aprender, primeiro você deve começar a compreender. Mas, como se pode aprender a compreender matemática? Em outras palavras, para usar a famosa frase, como aprender a aprender matemática? Este é o desafio e a aposta no ensino de matemática. E é aí que encontramos a segunda exigência relativa aos objetivos dos sistemas educativos em termos de "saberes a aprender", uma vez que se trata de levar em conta a desproporção crescente entre o que se pode aprender na escola e o que será necessário aprender no âmbito da vida profissional e nas mudanças prováveis de ofício.

Várias direções têm sido exploradas. Para evocá-las, vou simplesmente repetir as palavras que vocês ouviram ou leram milhares de vezes: aprender "métodos" (métodos para encontrar a equação, métodos de resolução, método de construção, métodos de demonstração), aprender a "resolver problemas" (esta foi imposta, inicialmente, na educação matemática a partir dos anos de 1970-1980 para a introdução de novos conceitos), substituir a aquisição de "competências" pelo conhecimento teórico ou conceitual. Mas, estas diferentes pistas permitem enfrentar o desafio da educação matemática para o qual o ensino de matemática tem enfrentado por quase 50 anos, desafio este que é o da compreensão da matemática por todos os alunos de um grupo de idade que vai do ensino fundamental ao médio? Voltarei a esta questão na conclusão. 


\section{A mudança tecnológica digital: uma nova situação para o ensino. Mas, o que contribui para o desenvolvimento da autonomia intelectual?}

Embora o trabalho de "inteligência artificial" remonte a década de 1960, é o desenvolvimento fulgurante dos computadores pessoais, em torno da década de 1980, que ampliaram esta revolução. Por outro lado, é nessa época que os primeiros programas construídos para fins explícitos de ensino apareceram, o mais representativo deles é Cabri Géomètre. Não é demais recordar as suas primeiras motivações: trata-se de impor o recurso às propriedades geométricas, por meio de instruções, usando termos matemáticos para construir figuras, contra uma prática de orientação puramente perceptual. Desde então, programas computacionais foram desenvolvidos em todos os domínios do ensino da matemática, o mais espetacular deles é o de construção de gráficos para todos os tipos de funções. E agora, os programas multiplicam-se também para introduzir álgebra no ensino fundamental. É, atualmente, um pouco como um novo Eldorado para o ensino de matemática.

De um ponto de vista cognitivo, os programas computacionais fornecem três grandes inovações, a mais fascinante delas é o poder de visualização que é estendido para todos os domínios do conhecimento. A segunda, é que eles constituem em um meio de transformação de todas as representações produzidos no monitor. Dito de outro modo, eles não são apenas instrumentos de cálculo, em que o potencial cresce de modo ilimitado, mas eles preenchem uma função de simulação e modelização que excede tudo o que se possa conceber "mentalmente" ou no modo manual gráfico. Enfim, a produção é quase imediata: um clique e o resultado apresenta-se no monitor! Os programas computacionais AUTOMATIZAM A PRODUÇÃO COGNITIVA DE REPRESENTAÇÕES SEMIÓTICAS. É isso que desperta o interesse e a inovação de um ponto de vista cognitivo e que explica o papel cada vez mais primordial que desempenham no ensino de matemática (cálculo, álgebra, análise, geometria), uma vez que tornam as atividades de matemática mais imediatamente acessível e mais fácil. No entanto, a contribuição pedagógica dos programas computacionais para o desenvolvimento da mente e da autonomia intelectual em matemática permanece, no mínimo, ambígua e incerta.

Tomemos o exemplo da visualização em matemática e, mais particularmente, em geometria. Os programas computacionais atuais oportunizam, não só construir as figuras, mas explorar transformações de figuras por um simples deslocamento de um "objeto": um ponto, um segmento, etc. Não somente preenchem, também, uma função heurística, mas possibilitam 
uma abordagem "experimental", ao menos quase experimental das relações e propriedades geométricas. A visualização será, assim, completamente "externalizada" e a cargo do instrumento utilizado. Certo! Mas, ainda permanece uma dupla dificuldade a ser superada:

- a primeira delas é a articulação com os enunciados, propriedades e teoremas sem os quais não existe prova matemática. Este ponto é importante, não somente de um ponto de vista cognitivo, mas também de um ponto de vista matemático. De fato, para a visualização no monitor são usados processos de discretização que não possibilitam visualizar a continuidade matemática e o infinito. $\mathrm{E}$ isto chama a atenção para a primeira exigência cognitiva no desenvolvimento da autonomia intelectual do aluno.

(5) A compreensão em matemática, aliás, como em todos os domínios do conhecimento, implica, sempre, na coordenação entre linguagem e um ou mais tipos de representações visuais.

- a segunda dificuldade é o olhar do aluno que vê o que se apresenta na interface do monitor, deixando-se, espontaneamente, levar pelo reconhecimento perceptivo das formas. Ora, o primeiro passo na aprendizagem da geometria é aprender a ver contra todo reconhecimento perceptiva das formas. Existe um paradoxo da visualização que repousa sobre a desconstrução dimensional das formas $2 \mathrm{D}^{5}$ que se impõem de imediato e de maneira estável ao olhar. E isto não pode ser feito com um programa computacional, nem com o reconhecimento de figuras elementares, nem a partir de conceitos. Mas, é preciso admitir que até o presente momento, a importância cognitiva da desconstrução dimensional das formas - que é o implícito, por excelência, dos conceitos e das definições geométricas - permaneceu totalmente ignorada no ensino e pelas teorias didáticas dominantes. Esta dificuldade alerta para outra condição cognitiva do desenvolvimento da autonomia intelectual:

(6) É preciso discernir as informações pertinentes em qualquer que seja a produção semiótica (enunciado, texto documentário, imagens, esquemas, gráficos, figuras geométricas) e organizar as informações pertinentes produzindo, por si mesmo, em outro tipo de representação semiótica além daquela que aparece no monitor.

\footnotetext{
${ }^{5}$ N. Trad. Duval utiliza o termo 2D para indicar figuras de dimensão 2, como por exemplo, a figura de um triângulo. Já o lado de um triângulo, um segmento de reta, é um elemento 1D (dimensão 1).
} 
Em matemática, esta segunda exigência é fundamental para a resolução de problemas. Ora, mesmo quando um ensino se organiza em função de resolução de problemas, mantém-se, ainda, a questão relevante de saber como cada aluno torna-se autônomo na resolução do problema.

As condições cognitivas de (1) a (6), exceto, talvez (2), constituem os elementos mínimos do que é uma autonomia completa. E é neste sentido que a matemática é, pedagogicamente, fundamental para o desenvolvimento das capacidades da mente.

\section{Conclusão: educação ou formação}

Para terminar, eu gostaria de chamar a atenção para duas abordagens contrárias às finalidades dos sistemas educativos na aquisição dos "saberes". A primeira, a qual eu resevei a palavra "educação" (paideia) está centrada no desenvolvimento individual e na autonomia intelectual. A segunda, para a qual a palavra "formação" se impôs, visa à aprendizagem de saberes necessários em proveito da atividade profissional. Estas duas abordagens correspondem, aliás, a dois períodos dos sistemas educativos, a do desenvolvimento da criança e do pré-adolescente ${ }^{6}$ e da orientação profíssional. É evidentemente necessário não confundir estes dois períodos. Os objetivos e as apostas dos sistemas educativos não podem ser os mesmos para cada um desses períodos para o devir adulto e à aquisição de "saberes". Ora, a explosão dos conhecimentos e das novas tecnologias, assim como as rupturas sociais que começaram a vir à luz desde os anos 1960, acarretaram duas grandes transformações nos sistemas educativos. Uma é o prolongamento da duração dos estudos para mais de 20 ou 22 anos. A outra, que está associada a esta duração, é a generalização da abordagem da aquisição dos saberes como um objetivo de formação em detrimento da educação, quer dizer, do desenvolvimento da autonomia intelectual. O sintoma mais forte desta generalização e

\footnotetext{
${ }^{6}$ Há o período da infância e da pré-adolescência em que cada indivíduo se torna ele mesmo, quer dizer, se apropria do funcionamento de todos os sistemas que vão permitir que aja em seu ambiente e de se integrar a sociedade: seu próprio corpo inicialmente, os instrumentos e aparelhos que constituem o ambiente técnico e tecnológico e também todos os sistemas semióticos de representação (a começar pela língua materna) que constituem a memória do pensamento humano e seu vetor de desenvolvimento. Estes são, aliás, aqueles em que a aquisição é mais longa e mais complexa. É tudo isso que o indivíduo deve aprender a "ter o comando" e é em função desta tomada de comando que cada indivíduo torna-se autônomo. Para a tomada de comando das transformações ligadas aos sistemas semióticos que constituem a atividade matemática, chamo de "gestos intelectuais".
} 
desta homogeneidade, que vai do ensino fundamental ao universitário, é o recurso à análise da aquisição dos saberes em termos de competência. Para compreendê-lo, lembremos três coisas que são alusivas a esta noção:

- ela esta diretamente ligada à organização do trabalho nas empresas, quer dizer, a uma especialização dos tipos de atividades;

- as competências são analiticamente definidas como os comportamentos de resposta ou o saber-fazer exigido para uma atividade particular. Em outras palavras, o tipo de atividade ou o tipo de conhecimento cuja aprendizagem é esperada ao final de um percurso escolar, é decomposto em comportamentos ou conceitos pré-requisitais. E, estes pré-requisitos, tornamse objetivos pontuais de ensino em escala anual;

- a generalização desta noção nos sistemas educativos faz-se com avaliação, uma vez que tomou uma importância cada vez maior nos sistemas educativos em detrimento de uma abordagem do ensino em termos de educação ${ }^{7}$.

Em face desta generalização da análise da aprendizagem dos saberes como um objetivo de formação e em termos de competência, qual pode ser o aporte da matemática para o desenvolvimento da autonomia intelectual de cada aluno, sem o qual, a individualização, portanto, necessária, do ensino, estará fadada ao fracasso? Esta questão pode parecer tanto mais paradoxal e provocadora quanto a matemática, que provoca dificuldades de compreensão, as quais não se encontram em outras disciplinas e que a grande maioria dos alunos não consegue superar ao longo do percurso escolar. De onde vêm estas dificuldades profundas e recorrentes de compreensão? E, sobretudo, sob qual aspecto introduzir a matemática para que cada aluno possa, rapidamente, experimentar a sua autonomia intelectual e tornar-se capaz de aprender a aprender por ele mesmo e, não somente resolver problemas, mas ele mesmo poder formulá-los?

Até o presente, ainda não se prestou atenção suficientemente ao fato de que a atividade matemática apresenta duas características cognitivas e epistemológicas que a diferencia de todos os outros tipos de conhecimento (Ver 2.2). Concretamente, isto quer dizer que a atividade matemática tem sempre duas faces.

\footnotetext{
${ }^{7}$ A avaliação faz-se, atualmente, em dois níveis que suscitam lógicas distintas: o nível micro-pedagógico das sequências didáticas em sala de aula e o nível macro-comparativo dos sistemas educativos que se impôs, recentemente, com as avaliações internacionais.
} 
Há a face exposta, a face dos conceitos e dos procedimentos matemáticos a serem utilizados para fazer matemática e para resolver problemas. Todo ensino é organizado segundo a ideia de que a compreensão está subordinada à aprendizagem destes conceitos e procedimentos e que, esta aprendizagem, deve ser feita em certa ordem matemática de construção. De um ponto de vista matemático, é absolutamente incontestável. Mas, de um ponto de vista cognitivo, a atividade matemática tem também uma face oculta. Esta face é a forma de trabalhar em matemática, quer dizer, "fazer" matemática. Ela é a mesma, quaisquer que sejam os domínios matemáticos e quaisquer que sejam os conceitos ou os procedimentos a serem utilizados; exige maneiras de raciocinar, de justificar, de ver, de organizar as informações, de compreender os enunciados que estão em contraposição com aqueles que são espontaneamente praticados em outros domínios exterior a matemática. Para compreender matemática e poder utilizá-la, é preciso tomar consciência das formas de ver, de raciocinar, de reconhecer e de organizar as informações pertinentes. Dito de outro modo, a autonomia intelectual situa-se, inicialmente, na face oculta da atividade matemática e não na face exposta da matemática.

Olhando-se, agora, o desenvolvimento da "educação matemática", a partir dos anos 1960-1970, pode-se observar que se fez em função de uma dupla de demanda institucional: uma ligada às reformas sucessivas relacionadas ao conteúdo dos programas em diferentes ciclos de ensino e, outra, muito mais desconcertante, da formação dos professores. E se pode, também, observar a tendência cada vez mais predominante para traçar objetivos e conteúdos de ensino de matemática em termos de formação e não em termos de educação. Ora, continuase a se ater a face exposta da matemática e a ignorar a face oculta no ensino fundamental comum a todos os alunos de um mesmo grupo de idade. Não é de se espantar que a matemática origine dificuldades profundas de compreensão para a grande maioria dos alunos, chegando mesmo a criar bloqueios, a não ser que se reduza o ensino de matemática à utilização de programas computacionais. Mas, qual é, então, a sua contribuição para a formação da mente? As pesquisas sobre o ensino da matemática, assim como a organização institucional do ensino até os 15-16 anos devem, de pronto, considerar a autonomia intelectual de cada aluno. É esta a condição para auxiliar os professores a preencherem esta nova função que será, talvez, a principal nos anos que virão, a de ser "guia" e "tutor", quer dizer, $\pi \alpha 1 \delta$ - $\alpha \gamma \omega \gamma \circ$ s. 


\section{Annexe \\ Mutations, en cours et à venir, des systèmes éducatifs. \\ Défis et repères des années 1960 aux années... 2030 ! 1}

Raymond Duval

Depuis leurs développements institutionnels au XIXème siècle, les systèmes éducatifs de chaque pays ont connu beaucoup de changements et de réformes dans l'organisation des curricula, des disciplines à enseigner, des outils pédagogiques utilisés, des examens et des évaluations. Mais, depuis les années 1960, on peut parler d'une véritable mutation, indépendante des décideurs, que ce soient des experts scientifiques, pédagogiques ou politiques. Et cela conduit à une rupture complète avec ce que l'Ecole primaire, le Collège et le Lycée ont été et sont encore.

\section{Défis actuels et à venir}

Cette rupture, on peut la voir sur les deux façades de tout système éducatif. D'un côté, il y a les exigences concernant les « savoirs » qu'un système éducatif doit faire acquérir. Et de l'autre, il y a l'enseignant et la classe.

1. Les exigences concernent les « savoirs » sont formulées en termes d'objectifs et de contenus à acquérir. Elles viennent des besoins de la société en matière d'éducation et de formation. Deux exigences semblent devenues primordiales. Et ce que je voudrais déjà souligner, c'est que ces deux exigences sont fortement en concurrence, d'un strict point de vue éducatif.

1.1 La première correspond à ce que j'appellerais la philosophie des enquêtes internationales PISA (Programme for International Student Assessment). Elle est parfaitement exprimée le Directeur de l'éducation de l'OCDE, responsable de l'évaluation du «niveau des élèves» à 15 ans, dans 65 pays. Je le cite dans un interview récent (Le Monde, samedi 30 Août

${ }^{1}$ Conférence donnée à la Faculté des Sciences Sociales et des Sciences de l'Education de l'Université de Chypre, le 20 novembre 2014. 
2014, en page 6) : «Le monde moderne se moque bien de ce que vous savez. Il s'intéresse en ce que vous savez en faire». Autrement ce ne sont plus les connaissances qui comptent, mais ce que vous pouvez mettre en pratique dans des situations concrètes. Vous reconnaîtrez là une approche des objectifs en termes de "savoir-faire » et de «compétences ». Cette exigence correspond à une double demande sociale concernant le monde du travail : qu'à la sortie les jeunes trouvent un emploi, et que toutes les entreprises ainsi que les services publics trouvent un personnel qualifié.

1.2 La deuxième exigence correspond à ce que j'appellerais le développement de la capacité à réfléchir et à apprendre par soi-même, contre l'inertie de ce que l'on 《sait » et contre l'anxiété devant l'inconnu d'une nouveauté, que traduisent des expressions comme « on n'a pas appris cela », « on n'a jamais vu», « comment faut-il faire? ». Ici,ce ne sont plus les savoirs et les savoir faire qui sont importants, mais ce qui touche à ce qu'on appelle d'un terme kantien, devenu aujourd'hui à la fois un but et... un moyen : l'«autonomie » de l'élève. Un slogan, apparu dans les années 1960-1970, la résume : « apprendre à apprendre ». On va alors mettre l'accent, entre autres choses, sur les méthodes, sur les procédures plus que sur les contenus. Cette exigence est évidemment plus ancienne. Mais elle est devenu impérative avec l'accélération des découvertes scientifiques, dont la notion de «progrès » donne maintenant une idée fausse, et aux transformations technologiques de tous les outils. Il en résulte un décalage grandissant entre, d'un côté, les connaissances enseignées et, de l'autre, les connaissances réelles qui sont en pleine expansion dans les différents domaines de la recherche et leurs applications dans tous les secteurs de l'activité professionnelle. Et ce décalage impose d'apprendre non seulement à l'école mais tout au long de la vie. Aujourd'hui, nous sommes dans une situation où l'on doit apprendre plus de connaissances tout au long de la vie, que l'on en a appris à l'école, au lycée ou même à l'université. C'est ainsi que le mot «formation» est venu relayer en quelque sorte le mot «éducation ». C'est à partir des années 1970 que l'on a commencé à organiser la «formation permanente », la «formation continue », la « formation des adultes ».

2. Il y a une remise en cause du couple \{classe, enseignant\} qui a été jusqu'à présent le couple moteur des systèmes éducatifs.

Elle a tout d'abord porté sur la manière d'enseigner. Cela a commencé à partir des années 1960, avec ce qu'on avait alors appelé la «massification de l'enseignement secondaire 
». Et cela s'est traduit par la remise en cause du cours magistral au profit des «méthodes actives ». Autrement dit, la fonction de l'enseignant ne pouvait plus être de transmettre directement des savoirs, mais celle d'organiser ou de « gérer » le travail des élèves en classe, pour qu'ils « construisent » les savoirs à acquérir et pour « donner du sens » à ces acquisitions.

Mais la remise en cause la plus radicale touche maintenant la classe, c'est à dire le principe même d'une organisation commune et uniforme du travail pour un groupe annuel de 20 à 40 élèves. Et il est important de rappeler que cette organisation commune ne se traduit pas seulement dans un programme de contenus à acquérir, mais dans l'élaboration de séquences d'activités et $\mathrm{d}$ «ingénieries didactiques » pour l'acquisition de chacun des contenus.

Ainsi, les recherches sur l'enseignement des mathématiques et sur la formation des enseignants, commencées dans le cadre IREM à l'occasion de la Réforme des mathématiques modernes, se sont surtout développées en ce centrant sur l'organisation du travail en classe. Or c'est ce principe même qui apparaît maintenant inadapté et dépassé. La disparition de la classe est inévitable, et prévisible pour ... 2030 ! C'est du moins la conclusion à laquelle un forum de 645 «spécialistes mondiaux de l'éducation» est arrivée (Le Monde, 10 octobre 2014, en page 10). Car il y a une tendance à «l'individualisation de l'enseignement», entraînant ipso facto des «contenus beaucoup plus individualisés» qui va supplanter toute organisation commune de l'enseignement.

Ce qui est important concernant cette tendance n'est pas de savoir si elle va ou nous conduire à une disparition partielle ou complète de la classe, mais de voir pourquoi les mutations technologiques de l'environnement, d'une part, et les échecs des systèmes éducatifs, d'autre part, imposent une individualisation de l'enseignement.

2.1 La mutation technologique la plus puissante est celle du « numérique » qui a universalisé dans tous les domaines l'action interactive avec un écran. Une commande réduite à un clic sur un menu ou au toucher d'une icône fait apparaître à l'écran les images ou les informations que l'on souhaite. Cette mutation a permis une double individualisation:

— Chacun peut avoir un accès individuel direct et immédiat à toutes les connaissances et tous les échanges possibles, depuis le développement d'Internet et des sites Web (19891994). 
- Chacun peut utiliser lui-même, sans réel apprentissage préalable, des outils de traitement de l'information (calculatrices, traitement de textes, logiciels de construction graphiques, de construction de figures, etc.).

Tout cela s'est imposé avec le développement des micro-ordinateurs (1970-1976), c'est à dire des ordinateurs personnels, qui court-circuitent la production à la main de textes et de dessins, production qui est coûteuse, limitée, et qui surtout, exige de très longs apprentissages.

2.2 Les limites et les échecs des systèmes éducatifs apparaissent d'abord à travers les échecs des élèves et la manière dont ils les vivent. Cela se manifeste par la démotivation et même la marginalisation d'une proportion de moins en moins négligeable d'élèves, surtout à partir du Collège. En France on parle des élèves qui « décrochent ». Mais cela se traduit non seulement à travers les enquêtes PISA, mais à travers toutes les évaluations, qu'elles soient faite à un niveau national ou à une échelle plus réduite, dans le cadre de recherches. Sans entrer dans la discussion de la pertinence des questions posées, et de la manière de les regrouper pour déterminer ce qui est acquis, toutes les enquêtes et toutes les évaluations peuvent être lues selon le principe de la bouteille à moitié vide ou à moitié pleine, ou de la bouteille au tiers pleine et au deux tiers vide. Tout dépend de ce que l'on veut conclure en ce qui concerne la réussite ou l'échec d'un système éducatif, ou celle d'une expérience d'enseignement.

\section{Repères pour une autre éducation : le cas exemplaire et crucial de l'enseignement des mathématiques}

J'ai évoqué les défis. Je voudrais maintenant indiquer quelques points de repères pour une éducation qui prépare les enfants et les jeunes à un monde en pleine transformation technologique, culturelle et sociétale. Pour cela je vais prendre l'exemple de l'enseignement des mathématiques. Pourquoi ce choix? Tout d'abord je travaille sur l'enseignement des mathématiques depuis la création des IREM en 1969 et, là, j'ai pu suivre de près, à la fois auprès des élèves et auprès des enseignants, les évolutions et les mutations que j'ai évoquées. Mais il y a deux autres raisons, plus sérieuses. Elles touchent les objectifs de tous les systèmes éducatifs. Les mathématiques jouent un rôle clé pour l'apprentissage des autres disciplines et, surtout, la compréhension des mathématiques est pédagogiquement fondamentale et 
irremplaçable pour le développement des capacités de l'esprit. C'est sur leur rôle de formation de l'esprit que je voudrais insister. Et, pour cela, je vais retenir deux aspects de la compréhension des mathématiques, en relation avec les défis évoqués.

2.3 Les mathématiques sont ce type de connaissance où :

— personne ne peut vraiment comprendre à votre place

— on ne peut jamais répéter ou reproduire ce qui a été appris, ce qu'on trouve sur un site, ou ce que les autres vous ont montré.

Cela signifie deux choses qui peuvent paraître troublantes d'un point de vue éducatif traditionnel.

(1) À la différence des autres connaissances, les connaissances mathématiques ne sont pas communicables. Plus exactement, on peut comprendre les résultats scientifiques sans avoir à faire les démarches scientifiques qui permettent de les découvrir. Mais les résultats mathématiques ne sont pas compréhensibles tant qu'on ne peut pas refaire soi-même les démarches qui y conduisent. En termes pédagogiques, cela veut dire que les explications des autres (enseignant ou élève) ne réussiront à vous faire comprendre. C'est seulement ce qu'on vous réussit à faire par soi-même, tout seul, qui permet de comprendre en mathématiques.

(2) Vous ne pouvez pas utiliser des connaissances mathématiques apprises (les formules, par exemple) pour résoudre des problèmes de la réalité ou des problèmes scientifiques si vous n'avez pas compris vous-mêmes comment on pense et on travaille en mathématiques.

Autrement dit, les mathématiques sont ce type de connaissance dont la pratique reste éminemment individuelle, même si elle permet une communication objective et scientifique, mais uniquement ... entre ceux qui comprennent déjà les mathématiques! Car si on ne comprend pas par soi-même, les mathématiques ne sont que du jargon, des suites de chiffres aveugles ou des graphiques en trompe-l'œil qui s'affichent sur des écrans. La compréhension en mathématiques s'inscrit dans cette exigence d'individualisation de l'enseignement (c'est-àdire d'un enseignement qui ne soit plus centré sur l'organisation du travail en classe), qui s'impose comme une nécessité pour tous les systèmes éducatifs. 
2.4 Les mathématiques c'est, dès le départ, l'expérience d'une autonomie intellectuelle complète. Pourquoi ? En mathématiques, il faut pouvoir faire par soi-même pour comprendre (supra 1.1). Or l'activité mathématique présente deux caractéristiques cognitives et épistémologiques qui la différencient de tous les autres types de connaissance et d'activité. Elle se développe indépendamment de toute source externe d'informations, uniquement en transformant des représentations produites dans les systèmes sémiotiques communs (langue naturelle et diagrammes) et dans les systèmes sémiotiques spécialisés (systèmes numériques, graphes, figures géométriques). Et, grâce à ces transformations de représentations sémiotiques, l'activité mathématique donne une puissance illimitée d'exploration, qui est créatrice de connaissances nouvelles. Autrement dit, la compréhension en mathématiques repose sur l'autonomie d'une activité qui est purement intellectuelle (ce qui ne signifie pas purement «conceptuelle») qu'il faut pratiquer soi-même. Cette autonomie, qui est l'autonomie de la pensée, se manifeste des deux manières suivantes :

(3) Vous n'avez plus besoin de demander à quelqu'un d'autre (enseignant ou élève) pour savoir si ce que vous trouvez est vrai ou non, ou si un résultat est juste ou faux. Vous pouvez expliquez comment faire pour obtenir un résultat et, aussi, pourquoi ça marche ou pourquoi ça ne peut pas marcher.

(4) Vous n'avez plus besoin de demander à quelqu'un d'autre « qu'est-ce qu'il faut faire ?» devant un problème à résoudre, vous prenez des initiatives pour chercher ce qui va conduire à la solution. Vous prenez confiance en votre capacité d'analyse et d'initiative face à quelque chose d'inconnu ou de nouveau

Cela peut se résumer dans le cercle didactique suivant. Pour apprendre, il faut d'abord commencer par comprendre. Mais comment peut-on apprendre à comprendre en mathématiques? Autrement dit, pour reprendre la formule célèbre, comment apprendre à apprendre en mathématiques ? C'est le défi et l'enjeu de l'enseignement des mathématiques. Et c'est là qu'on retrouve la deuxième exigence concernant les objectifs des systèmes éducatifs en matière de "savoirs à acquérir ". Car il s'agit de prendre en compte la disproportion croissante entre ce qu'on peut apprendre à l'école et ce dont aura besoin d'apprendre dans le cadre de la vie professionnelle et des changements de métier probables. 
Plusieurs directions ont été explorées. Pour les évoquer, je me contenterai de répéter les mots que vous avez entendus ou lus des milliers de fois : apprendre des "méthodes» (méthodes de mise équation, méthodes de résolution, méthode de construction, méthodes pour démontrer), apprendre à « résoudre des problèmes » (cela s'est d'abord imposé dans la didactique des mathématiques à parti des années 1970-1980 pour introduire de nouveaux concepts), substituer l'acquisition de «compétences » à celle de connaissances théoriques ou conceptuelles. Mais ces différentes pistes permettent-elles de relever le défi auquel l'enseignement des mathématiques se trouve confronté depuis près d'une cinquantaine d'années, défi qui est celui de la compréhension même des mathématiques par tous les élèves d'une classe d'âge, au Primaire et au Collège? Nous reviendrons sur cette question en conclusion.

\section{La mutation technologique du numérique : une situation nouvelle pour l'enseignement, mais quel apport pour le développement de l'autonomie intellectuelle ?}

Bien que les travaux d'« intelligence artificielle » remontent aux années 1960, c'est le développement fulgurant des ordinateurs personnels, vers les années 1980 qui a amorcé cette véritable révolution. C'est d'ailleurs vers cette période que les premiers les logiciels construits à des fins explicites d'enseignement, sont apparus. Le plus emblématique est Cabri géomètre. Il n'est pas inutile de rappeler ses premières motivations. Il s'agissait d'imposer le recours aux propriétés géométriques, via des instructions utilisant les termes mathématiques, pour construire des figures contre la pratique d'un guidage purement perceptif. Depuis, des logiciels ont été développés dans tous les domaines de l'enseignement des mathématiques, le plus spectaculaire concernant la construction de graphiques pour tous les types de fonctions. Et, maintenant, les logiciels se multiplient aussi pour introduire l'algèbre au collège. C'est actuellement un peu comme un nouvel Eldorado pour l'enseignement des mathématiques.

D'un point de vue cognitif, ces logiciels apportent trois innovations majeures. La plus fascinante est la puissance de visualisation qu'ils apportent dans tous les domaines. La seconde est qu'ils constituent un moyen de transformations de toutes les représentations produites à l'écran. Autrement dit, ils ne sont pas seulement un instrument de calcul dont la puissance croit de façon illimitée, mais ils remplissent une fonction de simulation et de modélisation, qui excède tout ce que l'on peut imaginer «mentalement » ou effectuer de manière graphico-manuelle. Enfin, la production est quasi immédiate : un clic, et le résultat 
est sur l'écran! Ces logiciels AUTOMATISENT LA PRODUCTION COGNITIVE DE REPRESENTATIONS SEMIOTIQUES. C'est ce qui en fait l'intérêt et l'innovation du point de vue cognitif. Et c'est ce qui explique le rôle de plus en plus primordial qu'ils jouent dans l'enseignement des mathématiques (calcul, algèbre, analyse, géométrie). Car ils rendent les activités mathématiques plus immédiatement accessibles et plus faciles. Mais leur apport pédagogique pour le développement des capacités de l'esprit et de l'autonomie intellectuelle en mathématiques reste pour le moins ambigu et incertain.

Prenons l'exemple de la visualisation en mathématiques, et plus particulièrement en géométrie. Les logiciels permettent maintenant non seulement de construire des figures, mais d'explorer les transformations de figures par simple déplacement d'un « objet»: un point, un segment, etc. Non seulement ils remplissent ainsi une fonction heuristique mais ils permettraient une approche " expérimentale », ou du moins quasi expérimentale des relations et des propriétés géométriques. La visualisation serait ainsi complètement « externalisée» et à la charge de l'outil utilisé. O.K. ! Il reste cependant une double difficulté à surmonter.

La première est l'articulation avec les énoncés de propriétés et de théorèmes sans lesquels il n'y a pas de preuve mathématique. Ce point est important, non seulement d'un point de vue cognitif, mais aussi d'un point de vue mathématique. En effet, la visualisation à l'écran repose sur des processus de discrétisation et elle ne peut pas visualiser pas la continuité mathématique et l'infini. Et cela permet d'attirer l'attention sur une première exigence cognitive pour le développement de l'autonomie intellectuelle de chaque élève.

(5) La compréhension en mathématiques, comme d'ailleurs dans tous les autres domaines de la connaissance, implique toujours la coordination entre le langage et un ou plusieurs types de représentation visuelles.

La deuxième difficulté est l'œil de l'élève qui regarde ce qui apparaît dans l'interface écran, en se laissant spontanément guider par la reconnaissance perceptive des formes à l'écran. Or le premier pas dans l'apprentissage de la géométrie est d'apprendre à voir contre toute reconnaissance perceptive des formes. Il y a un paradoxe de la visualisation géométrique qui repose sur la déconstruction dimensionnelle des formes 2D qui s'imposent d'emblée et de manière stable au regard. Et cela ne peut se faire ni avec un logiciel, ni avec des reconnaissances de figures élémentaires, ni à partir de concepts. Mais il faut admettre que 
jusqu'à présent, l'importance cognitive de la déconstruction dimensionnelle des formes — qui est l'implicite par excellence des concepts et des définitions géométriques — est restée totalement ignorée dans l'enseignement et par les théories didactiques dominantes. Cette deuxième difficulté attire l'attention sur une autre condition cognitive pour le développement de l'autonomie intellectuelle.

(6) Il faut pouvoir discerner les information pertinentes dans n'importe quelle production sémiotique (énoncé, texte documentaire, images, schémas, graphiques, des figures géométriques) et organiser les informations pertinentes en produisant soi-même une autre type de représentation sémiotique que celui qui s'affiche à l'écran.

En mathématiques cette deuxième exigence est fondamentale pour la résolution de problèmes. Or, même lorsqu'un enseignement est organisé en fonction de la résolution de problèmes, il reste la question redoutable de savoir comment rendre chaque élève autonome dans la résolution de problème.

Les conditions cognitives (1) à (6), à l'exception peut-être de (2), constituent les composantes minimale de ce qu'est une autonomie intellectuelle complète. Et c'est en ce sens que les mathématiques sont pédagogiquement fondamentales et irremplaçables pour le développement des capacités de l'esprit.

\section{Conclusion : éducation ou formation?}

Pour terminer, je voudrais attirer l'attention sur les deux approches contraires des finalités des systèmes éducatifs pour l'acquisition des «savoirs». La première, pour laquelle je réserverai le mot «éducation » (paideia) est centrée sur le développement individuel et sur l'autonomie intellectuelle. La seconde, pour laquelle le mot «formation» s'est imposé, vise l'acquisition des savoirs nécessaires à un profil d'activité professionnelle. Ces deux approches correspondent d'ailleurs à deux périodes dans tous les systèmes éducatifs, celle du 
développement de l'enfant et du préadolescent ${ }^{2}$ et celle de l'orientation professionnelle. Il est évidemment nécessaire de ne pas confondre ces deux périodes. Les objectifs et les enjeux des systèmes éducatifs ne peuvent pas être les mêmes pour chacune de ces deux périodes dans le devenir adulte et dans l'acquisition des « savoirs».

Or l'explosion des connaissances et des nouvelles technologies ainsi qu'avec les ruptures sociétales qui ont commencé à se faire jour depuis les années 1960, ont entraîné deux transformations majeures dans les systèmes éducatifs. L'une est l'allongement de la durée des études, bien au delà de 20 ou 22 ans. L'autre, qui lui est évidemment associée, est la généralisation de l'approche de l'acquisition des savoirs dans un but de formation au détriment du but de l'éducation, c'est à dire du développement de l'autonomie intellectuelle. Le symptôme de la plus fort de cette généralisation et de cette homogénéisation, qui va du primaire à l'université est le recours à l'analyse de l'acquisition des savoir en termes de « compétences ». Pour le comprendre, rappelons trois choses concernant cette notion.

— Elle est directement liée à l'organisation du travail dans les entreprises, c'est à dire à une spécialisation des types d'activités.

— Les compétences sont analytiquement définies comme les comportements de réponse ou les savoir-faire exigés pour une activité particulière. Autrement dit, le type d'activité, ou le type de connaissance, dont l'acquisition est attendue en fin d'un parcours scolaire, est décomposé en comportements ou en concepts prérequis. Et ces prerequis deviennent des objectifs locaux d'enseignement à l'échelle d'une année.

\footnotetext{
2 Il y a la période de l'enfance et de la préadolescence où chaque individu devient lui-même, c'est à dire s'approprie le fonctionnement de tous les systèmes qui vont lui permettre d'agir dans son environnement et de s'intégrer dans la société : son propre corps tout d'abord, les outils et les appareils qui constituent l'environnement technique et technologique, et aussi tous les systèmes sémiotiques de représentation (à commencer par la langue maternelle) qui constituent la mémoire de la pensée humaine et son vecteur de développement. Ce sont d'ailleurs ceux dont l'acquisition est la plus longue et la plus complexe. C'est tout cela que chaque individu doit apprendre à «prendre en main », et c'est en fonction de cette prise en main que chaque individu prend confiance en lui et devient autonome. Pour la prise en main des transformations de représentations liées aux systèmes sémiotiques, qui constituent l'activité mathématique, nous parlerons de « gestes intellectuels ».
} 
- La généralisation de cette notion dans les systèmes éducatifs s'est faite avec l'évaluation. Car celle-ci a pris une importance de plus en plus en grande dans les systèmes éducatifs, au détriment d'une approche de l'enseignement en termes d'éducation ${ }^{3}$.

Face à cette généralisation de l'analyse de l'acquisition des savoirs dans un but de formation et en termes de compétences, quel peut être l'apport des mathématiques pour le développement de l'autonomie intellectuelle de chaque élève, sans laquelle l'individualisation, pourtant nécessaire, de l'enseignement, restera vouée à l'échec ? Cette question peut paraître d'autant plus paradoxale, et provocatrice, que les mathématiques soulèvent des difficultés de compréhension qu'on ne rencontre pas dans les autres disciplines et que la grande majorité des élèves n'arrivent pas à surmonter tout au long de leur parcours scolaire. D'où viennent ces difficultés profondes et récurrentes de compréhension? Et, surtout, sous quel aspect introduire les mathématiques pour que chaque élève puisse très vite faire l'expérience de son autonomie intellectuelle, et qu'il devienne ainsi capable d'apprendre à apprendre par lui-même et, non pas seulement de résoudre des problèmes, mais de les formuler lui-même ?

Jusqu'à présent, on n'a pas encore suffisamment prêté d'attention au fait que l'activité mathématique présente deux caractéristiques cognitives et épistémologiques qui la différencient de tous les autres types de connaissance (ci-dessus, 2.2). Concrètement cela veut dire que l'activité mathématique a toujours deux faces.

Il y a la face exposée, celle concepts et des procédures mathématiques à utiliser pour faire des mathématiques et pour résoudre des problèmes. Tout l'enseignement est organisé sur l'idée que la compréhension est subordonnée à l'acquisition de ces concepts et de ces procédures, et que cette acquisition doit se faire selon un certain ordre mathématique de construction. Du point de vue mathématique, c'est absolument incontestable. Mais, d'un point de vue cognitif, l'activité mathématique a aussi une face cachée. Cette face, c'est la manière de travailler en mathématiques, c'est à dire de « faire» des mathématiques. Elle est la même, quels que soient les domaines mathématiques et quels que soient les concepts ou les

\footnotetext{
${ }^{3}$ L'évaluation se fait maintenant à deux niveaux relevant de logiques différentes : le niveau micro-pédagogique des séquences didactiques en classe, et le niveau macro-comparatif des systèmes éducatifs, qui s'est imposé plus récemment avec les évaluation internationales.
} 
procédures à utiliser. Elle exige des manières de raisonner, de justifier, de voir, d'organiser des informations, de comprendre des énoncés qui vont contre celles qui sont spontanément mises en cuvre en dehors des mathématiques. Pour comprendre les mathématiques et pouvoir utiliser des connaissances mathématiques, il faut avoir pris conscience des manières de voir, de raisonner, de reconnaître et d'organiser les informations pertinentes. Autrement dit, l'autonomie intellectuelle se situe d'abord dans la face cachée de l'activité mathématique, et non pas dans la face exposée des mathématiques.

Si on regarde, maintenant, le développement de l' « éducation mathématiques » depuis les années 1960-1970, on peut remarquer qu'il s'est fait en fonction d'une double demande institutionnelle : d'une part, celle liée aux réformes successives concernant le contenu des programmes dans les différents cycles, et d'autre part, celle beaucoup plus contraignante, de la formation des enseignants. Et on peut aussi observer la tendance de plus en plus prédominante à décrire les objectifs et les contenus de l'enseignement des mathématiques en termes de formation, et non pas en termes d'éducation. Car on continue à s'en tenir à la face exposée des mathématiques et à en ignorer la face cachée, aussi bien au Primaire qu'au Collège, c'est à dire dans les deux cycles d'un enseignement commun pour tous les élèves d'une même classe d'âge. Rien d'étonnant à ce que les mathématiques soulèvent des difficultés profondes de compréhension pour la grande majorité des élèves, allant jusqu'à créer des blocages. A moins, bien sûr, que l'on réduise l'enseignement des mathématiques à l'utilisation de logiciels ! Mais quel peut être alors leur apport pour la formation de l'esprit?

Les recherches sur l'enseignement des mathématiques, ainsi que l'organisation institutionnelle de l'enseignement jusqu'à 15-16 ans doivent d'abord prendre en compte l'autonomie intellectuelle de chaque élève. C'est la condition pour aider les enseignants à remplir cette nouvelle fonction qui sera peut-être leur fonction principale dans les années à venir, celle de « guide » et de « tuteur », c'est à dire de $\pi \alpha \iota \delta-\alpha \gamma \omega \gamma o s$. 